\title{
Sense and Sensation: Exploring the Interplay Between the Semiotic and Performative Dimensions of Theatre
}

\section{Erika Fischer-Lichte}

\section{The Semiotic and the Performative}

In the 1970s, when the so-called linguistic, or semiotic, turn took place in the humanities, it opened up new possibilities for theatre research. Up to then, its main self-understanding had been that of a historical discipline analogous to art history and the history of literature. But whereas these disciplines regarded the analysis and interpretation of works of art from past epochs as their main task, a comparable approach was not open to theatre studies. Performances of the past are no longer accessible; they are gone and lost forever. The ephemeral and transitory nature of performance, which the German playwright and theoretician Gotthold Ephraim Lessing referred to as early as the $18^{\text {th }}$ century as theatre's unique peculiarity, does not allow the analysis or interpretation of a past performance. It is possible to examine documents on a performance as well as the material traces left behind, such as the theatre building, stage sets, costumes, props, the text of a play, a score, reports or reviews on the performance, etc., but not the performance itself.

On the other hand, a performance, which was accessible, i.e. contemporary theatre, was not considered a suitable object of research. For while the literary scholar or the art historian is able to take recourse to the object of his study whenever it is needed and become absorbed in its details, contemplating it as long as he feels necessary, the performance is not at the theatre scholar's disposal in a comparable way. Because of its fleeting nature, any attempt to analyze it seems doomed to failure. Therefore, dealing with contemporary performances was left to theatre critics, while the scholar's object of interest was taken from theatre history.

By the 1970s, when theatre scholars began to question this distribution of labor and tried to develop methods of analyzing a performance despite its ephemeral nature, semiotics provided them with a set of tools. Semiotically speaking, a performance can be defined as a structured coherence of theatrical signs such as

\footnotetext{
Erika Fischer-Lichte is professor of theatre research and director of the newly founded Institute of Advanced Studies on Interweaving Theatre Cultures at the Freie Universitaet Berlin. She is past president of the International Federation for Theatre Research and a member of the Academia Europaea, the Academy of Sciences at Goettingen and the Berlin-Brandenburg Academy of Sciences. Her publications include The Semiotics of Theatre (German 1983, English 1992), History of European Drama and Theatre (German 1990, English 2002), Kurze Geschichte des deutschen Theaters (1993), The Show and the Gaze of Theatre (1997), Das eigene und das fremde Theater (1999), Aesthetische Erfahrung. Das Semiotische und das Performative (2001), Theatre, Sacrifice, Ritual: Exploring Forms of Political Theatre (2005), and The Transformative Power of Performance: A New Aesthetics (2008, German 2004 as Aesthetik des Performativen).
} 
scenic space, the bodily appearance of the actors, gestures, movements, language, sounds, music, and so on, and in this sense as a text-which does not mean the literary text of a play but a text made up of heterogeneous signs. Thus, semiotic methods could be applied to a performance. ${ }^{1}$

The central purpose of semiotic analysis was to search for possible meanings. It stemmed from the assumption that an unequivocal meaning can never be accorded to a performance - as it cannot to lyrics or a painting. It is ambiguous and polyvalent. Accordingly, it is open to most diverse processes of meaning generation. It is never the meaning of a gesture, action, scene, or the whole performance, but only one possible meaning from a generally large number of meanings generated with regard to the element in question. A semiotic analysis never strives to discover a unified meaning of performance or any one element of it. Even if one particular meaning is generated, this does not mean that all others are to be excluded. Rather, a choice is made with a view to the particular problem, question, hypothesis, or perspective under which the analysis is undertaken, for there is no such thing as a complete analysis. An analysis will always neglect elements of the performance which do not seem relevant in terms of the leading question of the analysis or which were simply not perceived at all, first and foremost because an analysis is a dynamic process of relating elements of the performance to each other as well as to all kinds of elements to which the analyst may refer. Theoretically, this process can never come to an end. Instead, the end, created by the analyst, is somewhat arbitrary. Although theatre semiotics is sometimes blamed for being static, reducing the full range of possible meanings to one meaning only, for demanding clarity and not allowing ambiguity or even contradiction, for demanding a unified meaning, all such reproaches are pointless. If such a result has occurred, it has not followed from any restrictions in the semiotic approach per se, but from an inadequate application of it.

Another reproach is that theatre semiotics ignores the particular materiality of theatrical signs. This does not hold true either, for a semiotic analysis always proceeds from the very materiality of the sign. However, it must be conceded that it is only considered with regard to its potential to serve as a sign - to produce meaning. This can, in fact, be seen as a certain limitation.

While the linguistic turn of the 1970s resulted in an understanding not only of theatre but quite generally of culture as a text made up of signs that have to be deciphered, the so-called performative turn of the 1990s brought forth another metaphor which, in some ways, is opposed to that of "culture as text" - the metaphor of "culture as performance." In theatre studies, this resulted in a shift of focus from the semioticity of a performance to its performativity. This shift promised to overcome the limitations of theatre semiotics. From the perspective of performativity the interest is less in what the acts, actions, and movements of the performers mean than in how they are perceived and experienced, how they affect the spectators, and what kind of impact they have on them. Therefore, the 
focus on performativity often requires another kind of approach, which considers these particular qualities of a performance as, for instance, a phenomenological approach might.

Whereas a semiotic approach concerns itself with the conditions under which meanings in a performance may emerge out of its performative processes and with the meanings that are conceivable and possible, a phenomenological approach focuses on the performative processes as such. While the semiotic approach asks, "What do the performative processes mean," the performative approach instead raises the question, "What do they $d o$ ?" Therefore a semiotic analysis of a performance considers what is happening on stage as a text made up of theatrical signs that are to be interpreted. Analyses concerned with its performativity proceed from the eventness of a performance and highlight those performative processes which result in something happening between actors and spectators-that the spectators become colored, even affected and moved by what the actors/performers do. Hence it follows that the semiotic and the performative dimensions of a performance are different, although inextricably intertwined. They offer two different perspectives on the same object, the performance, which, ultimately cannot be separated from each other, and if so, only temporarily for heuristic reasons. I shall substantiate my argument by referring to Frank Castorf's production of Trainspotting in the Volksbuehne am Rosa-Luxemburg-Platz Berlin in 1997.

\section{Trainspotting}

The production was a theatrical adaptation of Irvine Welsh's first and highly successful novel, Trainspotting, and its adaptation in 1996 for a film by Danny Boyle. Although it was presented in the Volksbuehne, a theatre building with a stage and an auditorium, it was not the given spatial arrangement that defined the relationship between actors/performers and spectators, for the auditorium was not used at all. Instead the performance took place on the stage, and the spectators were seated on scaffolding at the back of the stage. To take their seats, they had to cross the stage, where some lighting equipment was laid out. Those spectators who had already taken their seats watched later arrivals stumble across the stage, sometimes destroying parts of the "set" though the ushers at the Volksbuehne had asked the spectators to watch their step when walking over the lighting equipment. Here, the spatial arrangement was used in order to redefine the roles of the spectatorsobviously, the roles of spectator and actor were being played with. Whether they wanted to or not, the arriving spectators took on the roles of actors before the gaze of the spectators already seated. At the same time, the performance toyed with the conventions that mark the beginning of the performance. Did it start as soon as the first spectator had reached the scaffolding and started to watch others arriving, or when the first actor from the Volksbuehne entered the stage?

Right from the very beginning, the spectators were somewhat irritated about 
their own role in the performance. They were seated on the stage-i.e., the place reserved for the actors, and those arriving later, in fact, acted before the eyes of the others. They were not free to decide on their own whether to become actors or to remain spectators - as in some sort of "old-fashioned" audience participation-but rather in order to become spectators they first had to act as performers. Stumbling over a faintly lit stage and accidentally disconnecting lighting cables while sensing the eyes of others staring at them, at least in some cases, resulted in a feeling of insecurity and awkwardness. In this way, the very process of entering the stage space in order to participate in a performance caused irritation. People who arrived in order to act as spectators were suddenly forced into the role of actors, which some apparently hated while others enjoyed the experience.

Moving over the stage in order to reach one's place stimulated some to try and make sense of the whole procedure. What was the purpose of being made to appear on stage? Was it meant to point to the permanent change between the role of actor and performer we undergo in everyday life? Or did it hint at a particular involvement of the spectators in the story to be acted out later on by the Volksbuehne actors-perhaps in the sense of the old Tua res agitur? While for some the walk over the stage might have been experienced as somewhat destabilizing - having their clumsiness exposed to the gaze of others - the attempt to make sense of it surely contributed to a re-stabilization.

However, during the course of the performance, the spectators quickly recognized that having arrived at the scaffolding, taken their seats, and thus regained the role of spectator, they were by no means in a safe position. The four actors repeatedly provoked interaction with the spectators. One of the actors (Hendrik Arnst), a man of massive physical proportions playing the part of Frank, went up to one or another spectator from time to time, violently stamping the ground in a terrifying way, attacking her/him with aggressive remarks, bodily posture, or gestures. He abused a female spectator in the upper rows as "fucking bitch" and bellowed at her to stop her "idiotic gawping." The reactions of the spectators were different at each performance. One spectator who was attacked obviously felt threatened and moved back, terrified; he seemed to make himself smaller and in this way invisible, while the other spectators burst out laughing. This seemed to be a welcome pretext for the actor to launch another attack on the audience by shouting to them: "Shut up when the lady is going to speak!" whereupon the actress Kathrin Angerer, who played the part of Alison, took up her scolding aria on Frank, the "swine," a second time. At another performance, the man addressed by the actor tried to ignore the attack, and the audience did not laugh. This time, the actor went back to the playing area, and the actress began her aria anew. On one occasion, during the performance first mentioned, two spectators wanted to leave before the end and tried to sneak out unnoticed by the actors. One actor (Matthias Matschke) managed to run up to them before they could leave and took them to 
task. He interrogated them as to why they did not like the performance and asked them to rethink their decision. When they determinedly opened the door and left without saying a word, he sent after them the rudest abuse-which the audience cheered. In any case, whatever happened, the spectators had a hard time claiming the position of distanced observers. The production did its utmost to make them part of the performance. The spectators experienced themselves as being determined by the course of the performance as it was not in their power to prevent the actors from addressing them directly, thus turning them into actors of the play. At the same time they experienced themselves as being able to co-determine the course of the performance by their observable reactions. On the one hand, this provoked a number of physiological, energetic, affective, or motor states in the spectators concerned; on the other, it raised the question of how to make sense of this kind of forced audience participation.

\section{The bodily co-presence of actors and spectator}

One possible meaning might have been created by making the spectators experience and, as a consequence, recognize that a performance always comes into existence through the bodily co-presence of actors and spectators who assemble at a certain time and place in order to share a situation, a moment in time. While those who "act" - "real" actors or spectators in the position of actors - do something - move through the space, perform gestures, manipulate objects, speak and sing - the spectators perceive them and react.

It may well be the case that such reactions are at least partly internalimaginative and cognitive-i.e., purely mental processes. However, most of the reactions and responses can be perceived by the actors and the other spectators, e.g., giggling, laughing, shouting, yawning, snoring, sobbing, crying, eating, drinking, commenting on what is happening, getting up, running out, and slamming the doors. The perception of such responses, in its turn, results in further perceptible reactions. Whatever the actors do, it has an effect on the spectators; and whatever the spectators do, it has an effect on the actors and other spectators. It can be concluded from this situation that a performance only comes into being through the interactions between actors and spectators. Hence it follows that its course cannot be completely planned or predicted and is, in fact, different each night. It is kind of an autopoietic process, which is characterized by differing degrees of contingency. Anything that occurs in the course of a performance cannot be entirely foreseen at its beginning. Many elements emerge in the course of a performance as a consequence of certain interactions.

Of course, the actors on stage - and the director behind them - set the decisive preconditions for the progression of the performance-preconditions that are fixed by the process of mise-en-scène. As director, Castorf decided on the place of the performance - the stage - and the special kind of "opening ceremony" that turned 
most spectators into actors. And it was the actor Hendrik Arnst, for example, who decided when, how, and which of the spectators to address directly. Nonetheless, neither was in a position to control the course of the performance entirely. In the end, it is all participants together who generate the performance.

Therefore, the performance opens up the possibility for all participants to experience themselves in its course as subjects that are able to co-determine the actions and behavior of others, and whose own actions and behavior, in the same way, are determined by others. The individual participants - whether actor or spectator-experience themselves as subjects neither fully autonomous nor fully determined by others, subjects who accept responsibility for a situation which they take part in but have not created. This demonstrates that a theatre performance is also to be regarded as a social process in which different individuals and groups encounter, negotiate, and regulate their relationships in different ways.

This "interpretation" is backed up at the end of the performance. The actors tried hard to draw as much applause from the audience as possible in order to extend the performance. Moreover, they not only jumped and smiled and stretched out their arms, they also stopped spectators who tried to steal away in order to ask why they were leaving so soon, whether they did not like the performance, or to scold them for being so stingy with applause. They mingled with the spectators, shaking - or, in the case of female spectators, kissing - their hands, and said, "Thank you." In other words, they fraternized with the spectators and tried all means to prevent them from leaving, extending the play into a clearly social situation and thus prolonging the performance. At the end, the spectators decided on the conclusion of the performance: it was only over when the last spectator left. This kind of ending unmistakably stressed that the performance had come into being through the encounter of actors and spectators.

By playing with and exploiting some of the possibilities that are given with the bodily co-presence of actors and spectators, the interplay between the performative and the semiotic dimension was also highlighted. It was the bodily experience of such co-presence which led to transformations of the physiological, affective, energetic, and motor states of the spectators and, following from that, to the semiotic processes by which spectators tried to make sense of such an experience.

\section{The materiality of the performance}

This interplay was also decisive with regard to the materiality of the performance as will be shown with regard to spatiality and corporeality.

\section{The space}

The playing area on the stage was formed by an almost empty space. In the back, where the curtain usually hangs, and at the right side, there were screens on which film clips were shown as, for instance, pictures of a landscape in spring, filmed from the window of a moving train, or a documentary on Nico Icon, the singer of 
Velvet Underground. Music was played not only during the film and video clips, but also in all parts of the performance. It included recordings by Velvet Underground, Iggy Pop, and Lou Reed, as well as Karel Gott (a Czech-German pop-singer born in 1939, who mostly performs easy listening music with folksy tunes and themes), and Arnold Schoenberg. There were only a few objects on stage: an iron bed where Alison was positioned during the whole performance, a baby doll, a toilet bowl, a Union Jack, and, on the floor, the lighting equipment mentioned above, laid out as it is used on construction sites. With the exception of the lights on the floor, none of the objects were in the playing area as the spectators made their entrance but were pushed on or brought in later by the three male actors.

The atmosphere which dominated the space changed several times during the course of the performance. Right from the start the spectators sensed a particular atmosphere, which was brought about by the huge dimension of the whole stage space with the naked wall behind only faintly lit. This, and the lighting equipment on the floor at the front and center stage, was reminiscent of a construction site, an atmosphere that was felt to be unpleasant. The atmosphere changed when the actors made their entrance, when the bed was pushed in, or when the toilet bowl was brought on stage. It also changed when the film and video recordings were presented or when music filled the space. However the atmosphere changed, it was always felt to be something one could not escape.

As the philosopher Gernot Boehme has shown, atmosphere, although not bound to a particular place, pours into the space. It is not tied to the objects - or people-from which it seems to emanate or to those who enter the space and sense it physically. Usually, atmosphere is the first thing that spectators perceive, "tingeing" them and thus allowing for a very specific experience of the space. An experience such as this cannot be explained by taking recourse to the single elements in space - its extension, particular objects, sounds, smells, or anything else. For it is not these individual elements that create the atmosphere but the interplay between all of them which, in theatre productions, is usually carefully calculated. Boehme defines atmosphere as "spaces, in that they are tinged by the presence of objects, of human beings or environmental constellations. They are themselves spheres of the presence of something, its reality in space."2 The phrase "spheres of presence" describes a particular mode in which objects are perceived. Boehme explains the mode in which a thing appears in a particular way as present, as an "ecstasy of the object." Not only its colors, smells, or sounds are conceptualized as ecstasies - i.e., the so-called secondary qualities of a thing — but also its primary qualities such as extension and form. The ecstasy of things influences their environment; they attract, even demand attention - as do the iron bed or the toilet bowl-and they appear to those who perceive them as present in a particularly intense way. They force themselves into their field of attention.

Atmosphere contributes considerably to the creation of spatiality. Through 
the atmosphere, which seems to permeate the space and the things, the things and the space appear to the subject who enters or who is present while it changes, as present in a highly emphatic sense. Not only do they present themselves in their so-called primary and secondary qualities; moreover, in the atmosphere, they even invade the body of the perceiving subject - which is mostly experienced through light, smells, and sound. For the spectator is not confronted with an atmosphere, is not distanced from it; rather, s/he is surrounded by it, s/he is permeated by it. In this sense, atmosphere is something which is physically sensed.

On the other hand, an atmosphere is able to work on the perceiving subject in a particular way because for the subject it connects with particular meanings which contribute to the feelings of, for example, uneasiness, awkwardness, or threat. As a result, the subject will accord various meanings to the atmosphere which it senses. It may well be that the particular shade of the atmosphere sensed deviates from any that have already been experienced in one respect or other. Thus the subject is challenged to find a new meaning which will inform the experience of the atmosphere at a later date, when it is sensed again. Therefore, atmosphere appears as a kind of phenomenon in which the performative and the semiotic dimensions are intertwined in a way that it is hard to separate them - and possible only for heuristic reasons.

\section{The actor's body}

Because of the bodily co-presence of actors and spectators, corporeality plays an essential role in performances. In Trainspotting this was not only effective when one of the actors went up to a spectator and addressed him or her directly, intimidating by his mere physical presence. It dominated the performance right from the very first entrance of the actors. The three male actors/junkies sat down side by side and flung their words out in a way that what they said was almost not intelligible. Instead, how they uttered it had an immediate effect on the spectators. The rhythm of their "choric" speech, the volume, the particular quality of the voices, the harmony or disharmony of their voices, and most of all the energy of the speakers had a physiological, affective, and energetic effect on the spectators. It seemed as if a stream of energy emanated from the actors, transferred onto the spectators, and energized them in their turn. In a particular way and with a particular intensity the actors were experienced as PRESENT.

What was sensed here was the phenomenal body of the actors, not their semiotic bodies that represented the junkies. While up to now the semiotic body representing dramatic figures in performances has attracted and received much attention, the phenomenal body of actors and spectators, i.e., their bodily being-in-the-world, has only seldom come into view. This is all the more surprising since the phenomenal body and the semiotic body are inextricably bound to each other; it is possible to think of the phenomenal body without referring to the semiotic body but not the other way round. It seems productive to relate them to one another via the concept 
of embodiment. ${ }^{3}$ By embodiment I do not mean the process of lending one's own body temporarily to something mental (an idea, a concept, a meaning, or even a bodiless spirit), which needs a body to articulate itself and gain presence. Rather, the term embodiment aims at the bodily processes by which the phenomenal body generates itself as a particular body and at the same time generates specific meanings. Thus, the actor brings forth and presents his phenomenal body in a very specific way through a process of embodiment which is sometimes experienced as PRESENCE. At the same time, he produces a dramatic figure, for example, Frank. In the performance, PRESENCE and the dramatic figure do not exist outside the particular process of embodiment by which the actor brings them into existence; rather, they are brought forth by them.

\section{Perception and Production of Meaning}

As already indicated, this interplay of the semiotic and the performative dimensions has consequences for the processes of perception and meaning generation which the spectators perform. To perceive the body, the things, and the space in their specific presence does not mean to perceive them as meaningless. Instead, all of these phenomena are perceived as something. We are not dealing with a non-specific stimulus here, mere sensorial data, but with a perception of something as something. In my perception, the objects appear in their particular phenomenality - the iron bed as an iron bed or the toilet bowl as a toilet bowl. These objects signify that which they appear to be. Their self-referentiality, accordingly, is not to be described as the mediation of a given meaning or as a desemantization of a sign but as a process of a very particular kind of production of meaning. This process is performed as the perception of a phenomenon in its particular materiality, in its phenomenal being. Perceiving and the generation of meaning, in this case, are performed in and by the very same act. Meaning is brought forth by and in the act of perceiving. In other words, in this case we do not perceive something first and then - in an act of interpretation-attribute the meaning of something else to it. Rather, the act of perceiving something as something is performed at the same time as the process of producing its meaning as this particular phenomenal being. I call this kind of perception the order of presence.

From this, I distinguish quite another kind of perception and production of meaning, namely, the order of representation. To perceive the actor's physicality in its bodily being-in-the-world lays the foundation for the order of presence. To perceive it as a sign for a dramatic figure or another symbolic order establishes the order of representation. When Kathrin Angerer takes the baby doll in her arms and rocks it, the doll is perceived as a sign for Alison's baby. When the cloth on the floor is identified by the spectator as the Union Jack, it is perceived as a symbol of the United Kingdom. And when the actors take it from the floor and stuff it into the toilet bowl, the spectators will perceive this as a sign process to which different 
meanings may be attributed - whether as something the dramatic figures want to use for cleaning the toilet bowl or to express an act of contempt for what the flag stands for, or whether the director wanted to take a stance in political theatre or something else again. While the first order produces meanings regarding the phenomenal being of the perceived, the second order brings forth meanings which, in their sum total, strive to constitute the dramatic figure or another symbolic order.

During the performance of Trainspotting - as is usual in a performance - the spectator's perception oscillated between both orders of perception. For instance, as long as Hendrik Arnst communicated with the other actors, he was mainly perceived as Frank, although his very peculiar physicality drew the attention of the spectator to his phenomenal body from time to time, to his particular bodily being-in-theworld. When he went up to the scaffolding and addressed a spectator with abuse or threats, it was rather his bodily being-in-the-world that was perceived. In this way, the spectator's perception was forced to shift from perceiving the semiotic body to perceiving the phenomenal body and vice versa. The moment the shift took place, a rupture occurred, a discontinuity manifested itself. A state of instability came into being that placed the perceiving subjects between the two orders, transferred them into a state of inbetweenness, of liminality. ${ }^{4}$

Each shift, each instability, that comes up in the course of a performance causes the dynamics of the process of perception to take another turn. The more often a shift happens, the more often the perceiving subjects begin to wander between two worlds, between two orders of perception. They increasingly become aware of their inability to cause, steer, and control the shifts. They may consciously try to adjust their perception anew - to the order of presence or to the order of representation. Very soon, however, they will become aware that the shift takes place even if they do not intend it, that it simply happens, befalls them, that they are moved between the two orders without wanting or being able to prevent it. At that moment, the spectators experience their own perception as emergent, as withdrawn from their will and control, and yet as an action performed consciously. That is to say that the shift draws the attention of the perceiving subject to the process of perception itself as well as to its particular dynamics. At the moment of shift, the process of perception itself becomes conspicuous, thereby self-conscious, and in itself the object of perception. The perceiving subject begins to perceive itself as perceiving subject. That is a performative as well as semiotic process in itself and produces new meanings which, in turn, generate other meanings and so forth. In this way, the process of perception continuously takes another turn. What is perceived and what meanings are produced become less and less predictable. The perceiving subjects become aware that the meanings are not conveyed to them but that it is they themselves who produce them and that they could have generated quite a different set of meanings if the shift from one order to the other would have happened earlier, later, or less frequently. 


\section{The performance as event-aesthetic experience as liminal experience}

From these different kinds of interplay between the performative and the semiotic in performance it can be concluded that a performance cannot be considered a work of art but an art event. Since a performance comes into being by way of the interaction between actors and spectators, since it brings forth itself in and through an autopoietic process, it is impossible to label it a work. For when the autopoietic process comes to an end, the performance does not remain as its result; rather, the performance has come to an end, too. It is over and therefore irretrievably lost. It exists only as and in the process of its performance; it exists only as event.

The performance as event-contrary to the mise-en-scène-is unique and cannot be repeated. It is impossible for exactly the same constellation between actors and spectators to occur another time. As has been shown with reference to Trainspotting, the response of the spectators and their effect on the actors and other spectators will be different with each and every performance. A performance is also to be understood as an event in the sense that no one participant can completely control it, that it simply happens to them - particularly to the spectators. This holds true not only with respect to the consequences of the bodily co-presence of actors and spectators, but also regarding the particular presentness of the phenomena as well as the emergence of meaning. As explained above concerning the shift of perception, it also befalls the perceiving subjects and transfers them into a state of betwixt and between, into a state of liminality.

Moreover, the particular eventness of performance is characterized by a strange collapse of oppositions. The participants in a performance experience themselves as subjects who co-determine its course and, at the same time, are determined by it. They live through the performance as an aesthetic as well as a social process in the course of which relationships are negotiated, power struggles fought, communities established and dissolved. Through embodiment, the actors bring forth the body as phenomenal as well as semiotic, and produce presence as well as meaning. The spectators' perception follows the order of presence as well as that of representation. This is to say that what is traditionally held to be an opposition in Western cultures, which is grasped by pairs of dichotomous concepts - such as autonomous subject vs. subject determined by others, art vs. social reality, presence vs. representation, the performative vs. the semiotic - is not experienced in the mode of either-or but in that of an as-well-as in performance. The so-called oppositions collapse, the dichotomies dissolve.

The moment this happens, when one category can also be taken for another, our attention is attracted by the passage from one state to the other, by the instability, which, in its turn, is experienced as an event. In the space between these opposites, an interval opens up. The "betwixt and between" which Turner stresses with regard to ritual, ${ }^{5}$ thus becomes a privileged category for the aesthetics of performance. It 
points to the threshold between the spaces, to the state of liminality into which the performance transfers all those who participate in it.

Since such pairs of dichotomous concepts serve not only as tools for the description and cognition of the world but also as regulators for our actions and behavior, their destabilization not only upsets our perception of the world, ourselves, and others but also shatters the rules and norms that guide our behavior. Different frames can be deduced from the pairs of concepts, for instance, "This is theatre/art" or "This is a social or political situation." Such frames prescribe an adequate behavior in the situation they encompass. By letting opposite or only different frames collide, by thus allowing different, even completely opposite values and claims to stand side by side so that they are all valid while at the same time they annul each other, performances create liminal situations. They transport the spectators between all these rules, norms, orders - sometimes they even transfer them into a crisis.

The performance transfers the spectators into a state, which alienates them from their everyday life and from the norms and rules valid in it. Such a state can be experienced as a pleasure as well as a torment. The transformations undergone by the subjects can be most diverse. Mainly, they are temporary transformations, which last only for a limited time span in the performance. These include changes in the body's physiological, affective, energetic, and motor states, a well as changes in status such as those from the status of a spectator to that of an actor, or the building up of a community between actors and spectators or only among the spectators. Such changes take place during the performance and are perceptible; after the performance has come to an end, however, they usually do not continue. This marks the difference between liminal states in ritual and in artistic performance. While in a ritual the transformation undergone is irreversible and the change of status requires social acceptance, this is not the case in an artistic performance. From this, it follows that although both ritual and aesthetic experience can be defined as liminal experience, they are by no means the same. While in a ritual the state of liminality is the path to gain a new status or identity, in an artistic performance it is an end in itself. However, although aesthetic experience does not result in a socially accepted change of status and identity, it may well cause a change in the perception of reality, self, and others in individual participants which will also influence this individual's action and behavior. This applies not only to the artists involved but also to the spectators. In this sense, the event may result in a transformation of the participants, which can even outlast the end of the performance.

As has become evident, performances always have a performative and a semiotic dimension. Both interact constantly, so that it is sometimes impossible to distinguish one clearly from the other. However, in the mise-en-scène of a performance as well as in the perception of individual spectators there may be a tendency to privilege one over the other. For instance, particular staging strategies 
may be intended and applied that strive for privileging one dimension and for guiding the spectator's perception in such a way that either the performative mode outweighs the semiotic one or vice versa. Nonetheless, such guidance does not often prove successful, and spectators may respond in an unpredictable way. On the other hand, there will always be spectators who tend to privilege one dimension, even if the staging strategies privilege the other. Whatever they perceive, they will either first ask what it means or, on the contrary, try to get immersed in the atmosphere, respond to the stream of energy which emanates from an actor and allows them to be energized, and vibrate with rhythms set by the mise-en-scène, without asking for meanings. In both cases, the rather neglected dimension will also always be effective since, as we have seen, both are inextricably intertwined.

This has to be kept in mind when undertaking a performance analysis. It depends on the leading question, the main problem, or the initial hypothesis as to whether the analysis will consider both dimensions equally well or one more than the other. In any case, it is wise to consider both even if to a different degree and in differing depth. For we are never to forget that it is the particular interplay of the performative and the semiotic dimension which constitutes performance.

\section{Notes}

1. Cf. Erika Fischer-Lichte, The Semiotics of Theatre, tr. Jeremy Gaines and Dores L. Jones (Bloomington: Indiana U P, 1992).

2. Gernot Boehme, Atmosphaere. Essays zur neuen Aesthetik (Frankfurt a.M.: Suhrkamp, 1995) 33.

3. Regarding this concept see Thomas J. Csordas, Embodiment and Experience. The Existential Ground of Culture and Self (Cambridge: Cambridge U P, 1994) and Erika Fischer-Lichte, "EmbodimentFrom Page to Stage: The Dramatic Figure," Assaph: Studies in Theatre No. 16 (2000): 65-75.

4. Regarding the concept of liminality cf. Arnold van Gennep, The Rites of Passage (Chicago: Chicago U P, 1960, originally 1909) and Victor Turner, The Ritual Process: Structure and Anti-Structure (Chicago: Aldine, 1969).

5. Turner 95 . 
